\title{
Cardiorespiratory Fitness is a Strong Predictor of the Cardio-ankle Vascular Index in Hypertensive Middle-aged and Elderly Japanese Men
}

\author{
Kumpei Tanisawa ${ }^{1}$, Tomoko Ito ${ }^{1}$, Xiaomin Sun ${ }^{1}$, Ryoko Kawakami ${ }^{1}$, Satomi Oshima ${ }^{2}$, Yuko Gando ${ }^{2}$, \\ Zhen-Bo $\mathrm{Cao}^{2}$, Shizuo Sakamoto ${ }^{2,3}$ and Mitsuru Higuchi ${ }^{2,3}$ \\ ${ }^{1}$ Graduate School of Sport Sciences, Waseda University, Saitama, Japan \\ ${ }^{2}$ Faculty of Sport Sciences, Waseda University, Saitama, Japan \\ ${ }^{3}$ Institute of Advanced Active Aging Research, Waseda University, Saitama, Japan
}

\begin{abstract}
Aim: This study aimed to examine whether cardiorespiratory fitness (CRF) is associated with arterial stiffening, evaluated using the cardio-ankle vascular index (CAVI), independent of visceral fat (VF) in middle-aged and elderly Japanese men. We also examined whether the relationship between CRF and the CAVI is modified by age and/or hypertension.

Methods: The CAVI was determined in 157 Japanese men (age range, 30-79 years), including 96

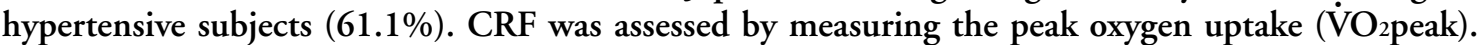
The subjects were divided into low- and high-CRF groups, and the VF area was assessed using magnetic resonance imaging.

Results: The $\dot{\mathrm{VO}}_{2}$ peak correlated with the CAVI following adjustment for age and body mass index in the middle-aged and elderly groups (all the subjects: $r=-0.285, p<0.001$; middle-aged: $r=-0.240$, $p=0.040$; elderly: $r=-0.225, p=0.049)$. VF also correlated with the CAVI $(r=0.230, p=0.004)$. A multiple linear regression analysis revealed that age $(\beta=0.406, p<0.001)$ and the $\dot{\mathrm{VO}}_{2 \text { peak }}(\beta=-0.186$, $p=0.015)$ were associated with the CAVI independently of VF and the mean blood pressure. Twoway ANCOVA adjusted for age demonstrated that the hypertensive individuals had higher CAVI values than the normotensive individuals in the low-CRF group, whereas no significant differences in the CAVI were observed in the high-CRF group ( $p$ for interaction $<0.05$ ).

Conclusions: In the present study, CRF was found to be associated with the CAVI, independent of age and VF, in hypertensive middle-aged and elderly Japanese men.
\end{abstract}

J Atheroscler Thromb, 2015; 22: 379-389.

Key words: Cardiorespiratory fitness, Visceral fat, Arterial stiffness, Hypertension,

Cardio-ankle vascular index

\section{Introduction}

Aging is associated with structural and functional changes in the vessel walls that subsequently result in decreased vascular elasticity and elevated arterial stiffness ${ }^{1,2)}$. Increased arterial stiffness is an independent

Address for correspondence: Mitsuru Higuchi, Faculty of Sport Sciences, Waseda University, 2-579-15 Mikajima, Tokorozawa,

Saitama 359-1192, Japan

E-mail: mhiguchi@waseda.jp

Received: March 20, 2014

Accepted for publication: September 16, 2014 predictive factor of mortality from cardiovascular disease $(\mathrm{CVD})^{3)}$. Although age-related declines in the arterial function are inevitable, numerous epidemiological studies have indicated that a high level of cardiorespiratory fitness (CRF) is associated with a reduced risk of $\mathrm{CVD}^{4,5)}$, which can, in part, be explained by the beneficial effects of a high CRF on arterial stiffness ${ }^{6}$. Moreover, a high CRF is associated with a low level of visceral fat (VF), independent of body mass index $(\mathrm{BMI})^{7)}$. Several studies have reported that arterial stiffness is closely associated with visceral obesity ${ }^{8,9)}$, indicating that the beneficial effects of a high CRF on 
the arterial function may be mediated via a decrease in VF. Therefore, it is debatable as to whether a high CRF level per se contributes to improving arterial stiffening independent of a high CRF-related decrease in VF.

Several previous studies have examined whether CRF is associated with arterial stiffening independent of indicators of body fatness, with inconsistent results. For example, Christou et al. ${ }^{10)}$ demonstrated that the association between CRF and arterial stiffening is lost after controlling for body fatness in terms of BMI, body fat percentage and waist circumference in healthy men. In contrast, Boreham et al. ${ }^{11)}$ indicated that CRF is independently associated with arterial stiffening after adjusting for age and skinfold thickness, a representative measure of subcutaneous fat (SF). However, these studies only measured simple anthropometric indices, and the VF-adjusted association between CRF and arterial stiffening has not been previously examined. Therefore, whether the beneficial effects of a high CRF value on arterial stiffening are independent of VF reduction remains unclear. Furthermore, most previous studies evaluated healthy young or middle-aged subjects, and trials focusing on older patients are relatively limited. Furthermore, previous analyses have reported that the age-related increase in arterial stiffness is particularly higher in older individuals with elevated blood pressure ${ }^{12,13)}$. Therefore, the relationships between the CRF and VF levels and arterial stiffening may differ according to age and the presence of hypertension.

Among a number of noninvasive measurements of arterial stiffness, the pulse wave velocity (PWV) has become widely used ${ }^{14)}$. However, the PWV is essentially influenced by blood pressure ${ }^{15,16)}$, and a previous study demonstrated that systolic blood pressure (SBP) is a major determinant of the brachial-ankle PWV ${ }^{17)}$. Therefore, it is difficult to assess arterial stiffness using the PWV in older individuals with high blood pressure. Recently, a new arterial stiffness index, the cardio-ankle vascular index (CAVI), was developed ${ }^{18,19)}$. The CAVI represents the degree of stiffness in the aorta and femoral and tibial arteries as a whole and is theoretically independent of blood pressure changes, as it is calculated based on the stiffness parameter $\beta$. Therefore, we speculate that the impact of CRF on arterial stiffness can be correctly evaluated by measuring the CAVI, even in older individuals with high blood pressure.

\section{Aim}

The aim of this study was to examine whether
CRF is associated with arterial stiffening, as evaluated according to the CAVI, independent of VF in middleaged and elderly Japanese men. We also aimed to examine whether the relationship between CRF and CAVI is modified by aging and the presence of hypertension.

\section{Methods}

\section{Subjects}

One hundred and fifty-seven middle-aged (age range: $30-64$ years, $n=77$ ) and elderly Japanese men $(65-79$ years, $n=80)$ participated in this study. The subjects had no history of diabetes or coronary heart disease and were free of other chronic diseases, such as cancer, chronic kidney failure, non-alcoholic steatohepatitis and autoimmune disorders. Thirty-three subjects $(21.0 \%)$ were treated with antihypertensive drugs, and 11 subjects $(7.0 \%)$ received lipid-lowering drugs. All participants were instructed not to take any medications on the morning of the day of the experiment. Ninety-six subjects $(61.1 \%)$ had hypertension, defined as a SBP of $\geq 140 \mathrm{mmHg}$ and/or diastolic blood pressure (DBP) of $\geq 90 \mathrm{mmHg}$ or the use of antihypertensive drugs. The current or former smoking status was recorded using a questionnaire, and daily alcohol intake was assessed using a brief selfadministered diet history questionnaire ${ }^{20)}$. All subjects provided their written informed consent prior to enrollment in the study, which was approved by the ethics committee of Waseda University. The study protocol was conducted in accordance with the Declaration of Helsinki.

\section{Anthropometric Characteristics}

The body weight and body fat percentage were measured using an electronic scale (Inner Scan BC-600, Tanita Inc., Tokyo, Japan), while height was measured using a stadiometer (YL-65, YAGAMI Inc., Nagoya, Japan). The BMI was calculated from the body weight and height measurements, and the VF and $\mathrm{SF}$ areas were measured on magnetic resonance imaging (MRI; Signa 1.5 T, General Electric Inc., Milwaukee, WI, United States). The imaging conditions included a T1-weighted spin-echo and axialplane sequence with a slice thickness of $10 \mathrm{~mm}$, repetition time of $140 \mathrm{~ms}$ and echo time of $12.3 \mathrm{~ms}^{21}$. Cross-sectional images of the umbilical region were obtained. During the scan, the subjects were asked to hold their breath for approximately 30 seconds after inhalation to reduce respiratory motion artifacts. The MRI scans were transferred to a personal computer in the Digital Imaging and Communications in Medi- 
cine file format, and the cross-sectional area of the VF at the umbilical region was determined using an image analysis software program (sliceOmatic 4.3 for Windows, TomoVision, Montreal, Quebec, Canada). In order to minimize interobserver variation, the same investigator performed all analyses, and the coefficient of variation was $0.4 \%$ for the cross-sectional areas of the umbilical region. The VF/SF ratio was calculated from the obtained measurements.

\section{Cardiorespiratory Fitness}

CRF was assessed according to a maximal graded exercise test using a cycle ergometer (Ergomedic 828E, Monark, Varberg, Sweden) and quantified as the $\dot{\mathrm{VO}}$ 2peak. The graded cycle exercise began at a workload of 45-90 W, which was then increased by $15 \mathrm{~W} /$ min until the subject could no longer maintain the required pedaling frequency of $60 \mathrm{rpm}$. The subject's heart rate and rating of perceived exertion were monitored every minute during exercise, and expired gas was collected during the incremental portion of the test. The $\mathrm{O}_{2}$ and $\mathrm{CO}_{2}$ concentrations were subsequently measured and averaged over 30 -second intervals using an automated gas analyzing system (Aeromonitor AE-300, Minato Medical Science, Tokyo, Japan). The highest $\mathrm{VO}_{2}$ value recorded during the exercise test was considered to be the $\dot{\mathrm{V}}$ 2peak $(\mathrm{mL} / \mathrm{kg} / \mathrm{min})$. The subjects were then subsequently divided into low- and high-CRF groups according to the median $\dot{\mathrm{VO}}$ 2peak value for each age group, as follows (in $\mathrm{mL} / \mathrm{kg} / \mathrm{min}$ ): 41.8 for 30-39 years, 36.4 for $40-49$ years, 38.9 for 50-59 years, 32.6 for 60-64 years, 30.5 for 65-69 years and 27.5 for $70-79$ years.

\section{Cardio-ankle Vascular Index}

The CAVI was measured with the VaSera VS-1500 device (Fukuda Denshi, Tokyo, Japan) using blood pressure and PWV measurements while monitoring electrocardiographic changes and heart sounds ${ }^{18,19,22)}$. The PWV was calculated by dividing the distance from the aortic valve to the ankle artery by the sum of the time between the aortic valve closing sound and the notch of the brachial pulse wave and the time between the increase in the brachial pulse wave and that of the ankle pulse wave. The CAVI was calculated using the following equation:

$$
\mathrm{CAVI}=\alpha \times\left[(2 \rho / \Delta P) \times \ln \left(P_{1} / P_{0}\right) \times \mathrm{PWV}^{2}\right]+b,
$$

where $P_{1}$ and $P_{0}$ are the SBP and DBP values, respectively, $\Delta P$ is $P_{1}-P_{0}$, PWV is the pulse wave velocity between the heart and ankle, $\rho$ is the blood density and $\mathrm{a}$ and $\mathrm{b}$ are constants. The day-to-day coefficient of variation for the CAVI was $2 \pm 1 \%{ }^{22)}$.

\section{Brachial Blood Pressure}

The brachial SBP and DBP values and mean blood pressure (MBP) at rest were measured using the oscillometric method (VaSera VS-1500) ${ }^{18)}$. All recordings were made in duplicate with the participant in the supine position.

\section{Collection and Analysis of Blood Samples}

Blood samples were collected between 0830 and 1100 after a 12-hour overnight fast and then centrifuged at $3,000 \times g$ for 15 minutes at $4{ }^{\circ} \mathrm{C}$. The serum and plasma were stored at $-80^{\circ} \mathrm{C}$ until the time of the analysis. The concentrations of total cholesterol, HDL cholesterol, LDL cholesterol, triglycerides and fasting glucose were determined using standard enzymatic techniques (BML, Inc., Tokyo, Japan). In addition, the glycated hemoglobin (HbAlc) levels were determined using the latex coagulation method (BML, Inc.), and the ratio of the LDL cholesterol level to the HDL cholesterol level (LDL cholesterol/HDL cholesterol) was calculated from the above measurements.

\section{Statistical Analysis}

All statistical analyses were performed using the SPSS version 21.0 software program (SPSS Inc., Chicago, IL, United States). Several variables were log transformed to obtain a normal distribution before the analysis. Student's $t$-test (for normally distributed variables) and the Mann-Whitney $U$ test (for nonnormally distributed variables) or chi-squared test (for categorical variables) were used to evaluate the differences between the middle-aged and elderly groups. The associations among the variables were determined using the Pearson correlation coefficient, and a partial correlation analysis adjusted for age was performed. Moreover, we examined the correlation between the CAVI and $\dot{\mathrm{VO}}$ peak values in each age group, and general linear models were used to assess differences in the slope and intercept of the regression line between the age groups. Furthermore, a multiple linear regression analysis was performed to determine factors that influence the CAVI. We first selected age, VF (or VF/ $\mathrm{SF}), \dot{\mathrm{VO}}$ peak and MBP as independent variables and the CAVI as the dependent variable. We next added the interaction terms " $\dot{\mathrm{VO}} 2$ peak $\times \mathrm{VF}$ (or $\dot{\mathrm{VO}} 2$ peak $\times$ $\mathrm{VF} / \mathrm{SF}$ )" and "نंO 2 peak $\times \mathrm{MBP}$ " to the first model. All the models were adjusted for alcohol consumption, current or former smoking, antihypertensive drug use, lipid-lowering drug use and variables that exhibited significant partial correlations with the CAVI values after adjustment for age and BMI. Multicollinearity among the variables was examined by calculating the variance inflation factor. The associations between the 
Table 1. Characteristics of the subjects $(n=157)$

\begin{tabular}{|c|c|c|c|}
\hline & Middle-aged & Elderly & $p^{*}$ \\
\hline \multicolumn{4}{|l|}{ Anthropometric characteristics } \\
\hline BMI $\left(\mathrm{kg} / \mathrm{m}^{2}\right)$ & $23.6 \pm 2.3$ & $23.5 \pm 2.4$ & 0.710 \\
\hline Body fat (\%) & $19.6 \pm 4.5$ & $21.0 \pm 4.7$ & 0.069 \\
\hline $\mathrm{VF} / \mathrm{SF}$ & $1.08 \pm 0.61$ & $1.33 \pm 0.71$ & 0.022 \\
\hline$\dot{\mathrm{V}} \mathrm{O}_{2 \text { peak }}(\mathrm{mL} / \mathrm{kg} / \mathrm{min})$ & $37.3 \pm 6.7$ & $29.1 \pm 5.4$ & $<0.001$ \\
\hline \multicolumn{4}{|l|}{ Vascular characteristics } \\
\hline $\mathrm{SBP}(\mathrm{mmHg})$ & $134.7 \pm 15.8$ & $142.2 \pm 14.1$ & 0.002 \\
\hline $\mathrm{DBP}(\mathrm{mmHg})$ & $89.1 \pm 10.4$ & $90.8 \pm 9.0$ & 0.273 \\
\hline Total cholesterol (mg/dL) & $209.2 \pm 35.5$ & $215.3 \pm 30.5$ & 0.248 \\
\hline HDL cholesterol (mg/dL) & $57.0(50.0-68.8)$ & $62.0(52.0-68.0)$ & 0.307 \\
\hline LDL cholesterol (mg/dL) & $121.4 \pm 30.6$ & $124.3 \pm 27.6$ & 0.542 \\
\hline LDL cholesterol/HDL cholesterol & $2.11 \pm 0.67$ & $2.11 \pm 0.70$ & 0.932 \\
\hline Triglycerides (mg/dL) & $94.5(67.3-125.0)$ & $83.0(65.0-118.0)$ & 0.361 \\
\hline Fasting glucose (mg/dL) & $93.9 \pm 9.3$ & $98.0 \pm 8.3$ & 0.004 \\
\hline HbA1c (\%) & $4.93 \pm 0.25$ & $5.02 \pm 0.28$ & 0.048 \\
\hline \multicolumn{4}{|l|}{ Confounders } \\
\hline Alcohol consumption (g/day) & $17.4(5.2-38.4)$ & $21.7(6.4-43.4)$ & 0.459 \\
\hline Current or former smoking status (\%) & 42.9 & 52.5 & 0.264 \\
\hline Hypertension (\%) & 50.6 & 71.3 & 0.009 \\
\hline
\end{tabular}

BMI: body mass index; VF: visceral fat; SF: subcutaneous fat; VF/SF: ratio of visceral fat to subcutaneous fat; $\dot{V}_{2}$ peak: peak oxygen uptake; SBP: systolic blood pressure; DBP: diastolic blood pressure; MBP: mean blood pressure; CAVI: cardio-ankle vascular index; LDL cholesterol/HDL cholesterol: ratio of LDL cholesterol to HDL cholesterol; HbA1c: glycated hemoglobin. The data are presented as the mean \pm SD or median (interquartile range) and were analyzed using Student's $t$-test (for normally distributed variables), Mann-Whitney's $U$ test (for non-normally distributed variables) or the chi-squared test (for categorical variables). Boldface text indicates significance $(p<0.05) .{ }^{*}$ Middle-aged vs. elderly.

fitness group, presence of hypertension and CAVI values were assessed using a two-way ANCOVA adjusted for age. A post hoc test with Bonferroni correction was employed to identify significant differences between mean values if a significant interaction was identified. All measurements and calculated values are presented as the mean $\pm S D$ (for normally distributed variables), median (interquartile range) (for non-normally distributed variables) or adjusted mean \pm SE (for the ANCOVA results), with statistical significance set at a $p$ value of $<0.05$.

\section{Results}

The characteristics of the subjects are presented in Table 1. The elderly group had lower $\dot{\mathrm{V}} \mathrm{O}_{2}$ peak values and higher VF, VF/SF, CAVI, fasting glucose and HbA1c values than the middle-aged group $(p<0.05)$. The proportions of subjects with hypertension and a history of antihypertensive drug use were significantly higher in the elderly group than in the middle-aged group $(p<0.05)$.

Table 2 shows the correlations between the CAVI values and the other variables. The cross-sectional analysis indicated that the CAVI was positively correlated with age, VF, VF/SF, SBP, DBP, MBP, fasting glucose and $\mathrm{HbAlc}$ and negatively correlated with the $\dot{\mathrm{VO}}$ ppeak $(p<0.05)$. The CAVI was also found to be significantly correlated with VF, VF/SF, $\dot{V O}_{2}$ peak, SBP, $\mathrm{MBP}$ and HbA1c following adjustment for age and 
Table 2. Correlations between the CAVI and other variables

\begin{tabular}{|c|c|c|c|c|}
\hline \multirow[t]{2}{*}{ Variables } & \multicolumn{2}{|c|}{ CAVI } & \multicolumn{2}{|c|}{$\begin{array}{c}\text { CAVI } \\
\text { (age- and BMI-adjusted) }\end{array}$} \\
\hline & $r$ & $p$ & $r$ & $p$ \\
\hline Age (years) & 0.663 & $<0.001$ & & \\
\hline BMI $\left(\mathrm{kg} / \mathrm{m}^{2}\right)$ & -0.100 & 0.214 & & \\
\hline Body fat (\%) & 0.088 & 0.272 & 0.070 & 0.386 \\
\hline $\mathrm{VF}\left(\mathrm{cm}^{2}\right)$ & 0.221 & 0.006 & 0.230 & 0.004 \\
\hline $\mathrm{SF}\left(\mathrm{cm}^{2}\right)$ & -0.098 & 0.224 & -0.022 & 0.785 \\
\hline $\mathrm{VF} / \mathrm{SF}$ & 0.305 & $<0.001$ & 0.210 & 0.009 \\
\hline$\dot{\mathrm{VO}}$ 2peak (mL/kg/min) & -0.553 & $<0.001$ & -0.285 & $<0.001$ \\
\hline $\mathrm{SBP}(\mathrm{mmHg})$ & 0.369 & $<0.001$ & 0.177 & 0.028 \\
\hline $\mathrm{DBP}(\mathrm{mmHg})$ & 0.282 & $<0.001$ & 0.132 & 0.102 \\
\hline MBP (mmHg) & 0.411 & $<0.001$ & 0.214 & 0.007 \\
\hline Total cholesterol (mg/dL) & 0.133 & 0.100 & -0.063 & 0.438 \\
\hline HDL cholesterol (mg/dL) & 0.093 & 0.251 & -0.124 & 0.126 \\
\hline LDL cholesterol (mg/dL) & 0.077 & 0.339 & -0.001 & 0.992 \\
\hline LDL cholesterol/HDL cholesterol & -0.008 & 0.920 & 0.059 & 0.465 \\
\hline Triglycerides (mg/dL) & 0.019 & 0.811 & 0.094 & 0.246 \\
\hline Fasting glucose (mg/dL) & 0.352 & $<0.001$ & 0.127 & 0.120 \\
\hline HbA1c (\%) & 0.383 & $<0.001$ & 0.224 & 0.006 \\
\hline
\end{tabular}

BMI: body mass index; VF: visceral fat; SF: subcutaneous fat; VF/SF: ratio of visceral fat to subcutaneous fat; $\dot{V}_{2}$ peak: peak oxygen uptake; SBP: systolic blood pressure; DBP: diastolic blood pressure; MBP: mean blood pressure; CAVI: cardio-ankle vascular index; LDL cholesterol/HDL cholesterol: ratio of LDL cholesterol to HDL cholesterol; HbA1c: glycated hemoglobin. The data are presented as Pearson's correlation coefficients. The HDL cholesterol and triglyceride levels were log transformed for the analysis. Boldface text indicates significance $(p<0.05)$.

BMI $(p<0.05)$.

In order to evaluate the influence of age on the relationship between the CAVI and CRF, we analyzed the correlation between the CAVI and $\dot{\mathrm{VO}}$ 2peak in each age group (Fig. 1). In the middle-aged and elderly groups, the CAVI values were negatively correlated with the $\dot{\mathrm{VO}}$ 2peak values $(r=-0.326, p=0.003$ and $r=-0.361, p=0.001$, respectively). These correlations remained significant after adjusting for age and BMI $(r=-0.240, p=0.040$ and $r=-0.225, p=0.049$, respectively). The slope of the regression line did not differ significantly between the age groups $(p=0.603)$, whereas the intercept did $(p<0.001)$.

In order to determine independent predictors of the CAVI, we performed a multiple linear regression analysis with the CAVI as the dependent variable (Table 3). In model 1, age was found to be most strongly associated with the CAVI after adjusting for the HbA1c level, alcohol consumption, current or former smoking, antihypertensive drug use and lipidlowering drug use $(p<0.001)$. Furthermore, the $\dot{\mathrm{V}} \mathrm{O}_{2}$ peak was also identified to be a significant predictor of the CAVI $(p=0.015)$, whereas VF and MBP were not shown to be associated with the CAVI, inde- pendent of age and $\dot{\mathrm{V}}$ 2peak $(p=0.104$ and $p=0.182$, respectively). In order to examine the synergic effects between the $\dot{\mathrm{VO}} 2$ peak, VF and MBP values on the CAVI, we further added the interaction terms "VO${ }_{2}$ peak $\times$ VF" and "ن을 Model 2 showed a significant interaction between $\dot{\mathrm{VO}} 2$ peak and MBP $(p=0.020)$, whereas no such interactions were observed between $\dot{\mathrm{VO}}$ 2peak and $\mathrm{VF}$ $(p=0.475)$. When VF/SF and the interaction term "VO 2 peak $\times$ VF/SF" were entered into the model instead of VF and " $\dot{\mathrm{VO}}$ 2peak $\times \mathrm{VF}$ ", VF/SF and the interaction term "VOO 2 peak $\times$ VF/SF" were not found to be independently associated with the CAVI, whereas the associations between the other independent variables and the CAVI did not change (age: $\beta=0.464, p<0.001$; $\dot{\mathrm{VO}}{ }_{2}$ peak: $\beta=-0.178, p=0.018$; VF/SF: $\beta=0.068, p=$ 0.265 ; MBP: $\beta=0.089, p=0.148$; $\dot{\mathrm{VO}}_{2}$ peak $\times \mathrm{VF} / \mathrm{SF}$ : $\beta=-0.094, p=0.125 ; \mathrm{VO}_{2}$ peak $\times \mathrm{MBP}: \beta=-0.133, p=$ 0.020 ; model $r^{2}=0.620$ ).

A multiple linear regression analysis indicated a significant interaction between $\dot{\mathrm{VO}}_{2}$ peak and $\mathrm{MBP}$, suggesting that blood pressure modifies the relationship between CRF and the CAVI. We thus compared the CAVI values between the low- and high-CRF 


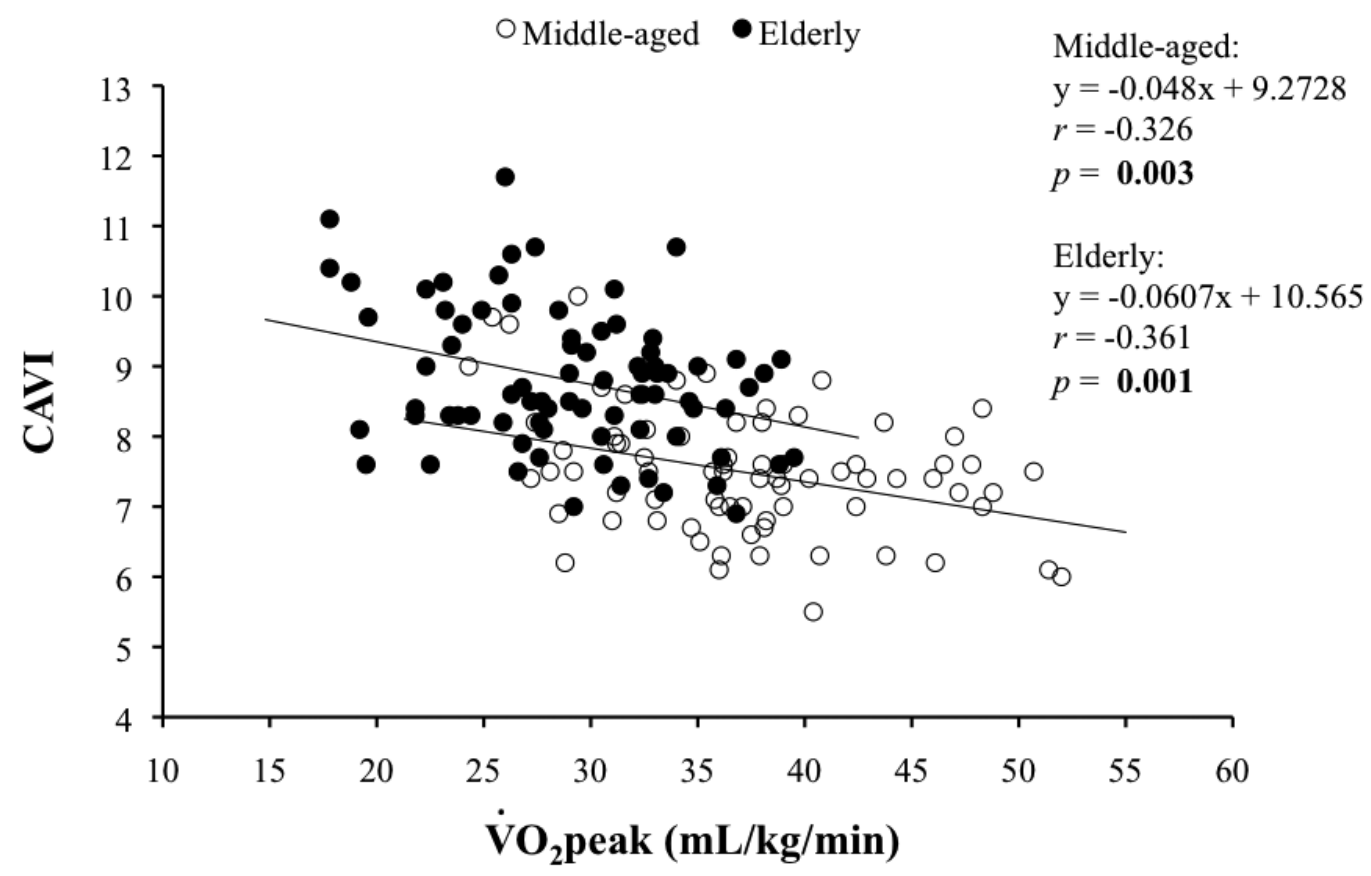

Fig. 1. Association between the CAVI and $\dot{\mathrm{VO}} 2$ peak values in the middle-aged and elderly Japanese men. Boldface text indicates significance $(p<0.05)$.

Table 3. Multiple linear regression analysis with the CAVI as the dependent variable

\begin{tabular}{|c|c|c|c|}
\hline Independent variables & $\beta$ & $t$ & $p$ \\
\hline \multicolumn{4}{|l|}{ Model 1} \\
\hline Age (years) & 0.406 & 5.425 & $<0.001$ \\
\hline$\dot{\mathrm{VO}}$ 2peak $(\mathrm{mL} / \mathrm{kg} / \mathrm{min})$ & -0.186 & -2.458 & 0.015 \\
\hline $\mathrm{VF}\left(\mathrm{cm}^{2}\right)$ & 0.135 & 1.635 & 0.104 \\
\hline MBP (mmHg) & 0.085 & 1.342 & 0.182 \\
\hline \multicolumn{4}{|l|}{ Model 2} \\
\hline Age (years) & 0.468 & 6.085 & $<0.001$ \\
\hline$\dot{\mathrm{VO}}$ 2peak $(\mathrm{mL} / \mathrm{kg} / \mathrm{min})$ & -0.158 & -2.089 & 0.039 \\
\hline $\mathrm{VF}\left(\mathrm{cm}^{2}\right)$ & 0.127 & 1.568 & 0.119 \\
\hline MBP (mmHg) & 0.069 & 1.099 & 0.274 \\
\hline$\dot{\mathrm{VO}}_{2 \text { peak }} \times \mathrm{VF}$ & -0.042 & -0.716 & 0.475 \\
\hline$\dot{\mathrm{VO}}$ 2peak $\times \mathrm{MBP}$ & -0.136 & -2.358 & 0.020 \\
\hline
\end{tabular}

CAVI: cardio-ankle vascular index; $\dot{\mathrm{V} O}$ 2peak: peak oxygen uptake; VF: visceral fat; MBP: mean blood pressure; $\beta$ : Standardized coefficient. The model was adjusted for BMI, MBP, HbA1c, alcohol consumption, current or former smoking, antihypertensive drug use and lipid-lowering drug use. Model 1, $r^{2}=0.593(p<0.001)$; Model $2, r^{2}=0.614(p<0.001)$. Boldface text indicates significance $(p<0.05)$.

groups according to the presence of hypertension. The two-way ANCOVA adjusted for age detected a significant interaction between the CRF group and the presence of hypertension on the CAVI (Fig. 2). In the low-CRF group, the hypertensive individuals had higher CAVI values than the normotensive individuals $(p<0.05)$, whereas no differences in the CAVI values were observed between the hypertensive and normotensive individuals in the high-CRF group. Furthermore, although the hypertensive individuals in the high-CRF group had lower CAVI values than those in the low-CRF group, no differences in blood pressure (SBP, DBP and MBP) were noted between the CRF groups (Table 4). Because the CAVI values differed 


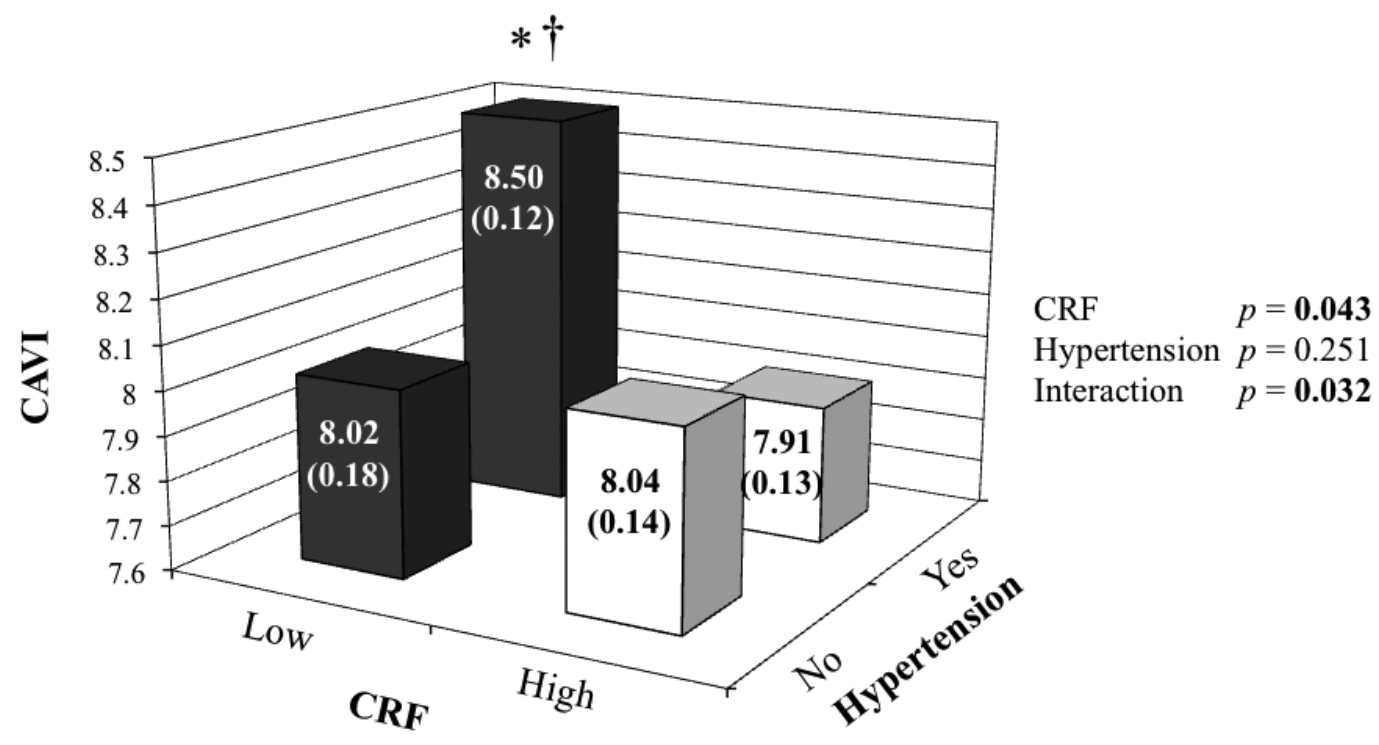

Fig. 2. Associations between the CRF group, presence of hypertension and CAVI values. The data are presented as adjusted means (SE) and were analyzed using a two-way analysis of covariance adjusted for age. Boldface text indicates significance $(p<0.05) .{ }^{*} p<0.05$ vs. low CRF among the hypertensive subjects. ${ }^{\dagger} p<0.05$ vs. normotension within the same CRF group.

between the CRF groups only among the hypertensive individuals, we additionally examined the correlation between the $\dot{\mathrm{V}} \mathrm{O}_{2}$ peak and CAVI values in the normotensive group. Consequently, the $\dot{\mathrm{V}} \mathrm{O}_{2}$ peak was found to be correlated with the CAVI in the normotensive individuals $(r=-0.430, p=0.001)$; however, the association was lost after adjusting for age $(r=-0.041, p=$ 0.757).

\section{Discussion}

In the present study, we demonstrated that CRF is a significant predictive factor of the CAVI, independent of age and VF, in middle-aged and elderly men (Table 3). Furthermore, the subsequent analysis demonstrated that the CRF values were robustly associated with the CAVI values only in the hypertensive individuals, without affecting blood pressure (Fig. 2). This is the first study to show that a high CRF is associated with reduced arterial stiffness, regardless of the VF area and blood pressure, in hypertensive middle-aged and elderly men.

To our knowledge, Lindholm et al. ${ }^{23)}$ conducted one of the few studies to investigate the relationship between CRF and the CAVI. They reported that the annual decline in maximal oxygen uptake $\left(\dot{\mathrm{VO}}_{2} \max \right)$ during a 13-year follow-up was significantly correlated with the CAVI after the follow-up period. However, the authors failed to detect a significant association between the $\dot{\mathrm{V}} \mathrm{O}_{2}$ max and CAVI independent of age in their cross-sectional observations. Because the CRF level inevitably declines with age ${ }^{24,25)}$, the relationship between CRF and the CAVI may be strongly mediated by aging. Nevertheless, in the present study, the correlation between the $\dot{\mathrm{VO}} 2$ peak and CAVI remained significant, even after adjustment for age (Table 2), suggesting that a high CRF has positive effects on the CAVI via several mechanisms, excluding aging.

Moreover, a negative relationship between CAVI and $\dot{\mathrm{V}}$ 2peak was observed among the middle-aged and elderly subjects in the present study, suggesting that a high CRF has a beneficial effect on arterial stiffening regardless of age (Fig. 1). However, the significant difference in the intercept of the regression line between the middle-aged and elderly groups, as shown in Fig. 1, indicates that the effect of aging on the CAVI certainly remains, even in subjects with high CRF levels. We should note that the age-related increase in the CAVI is not completely abolished by maintaining a high CRF level.

Previous studies have reported that the presence of hypertension accelerates the age-related increase in arterial stiffness in older individuals ${ }^{12,13)}$. We found it interesting that the CRF values were strongly associated with the CAVI values in the hypertensive individuals (Fig. 2). Inconsistent with our finding, Kraft et al. ${ }^{26)}$ reported that arterial stiffness, as assessed using the PWV, did not differ between the high- and low- 
Table 4. Associations between the CRF group, presence of hypertension and characteristics of the subjects

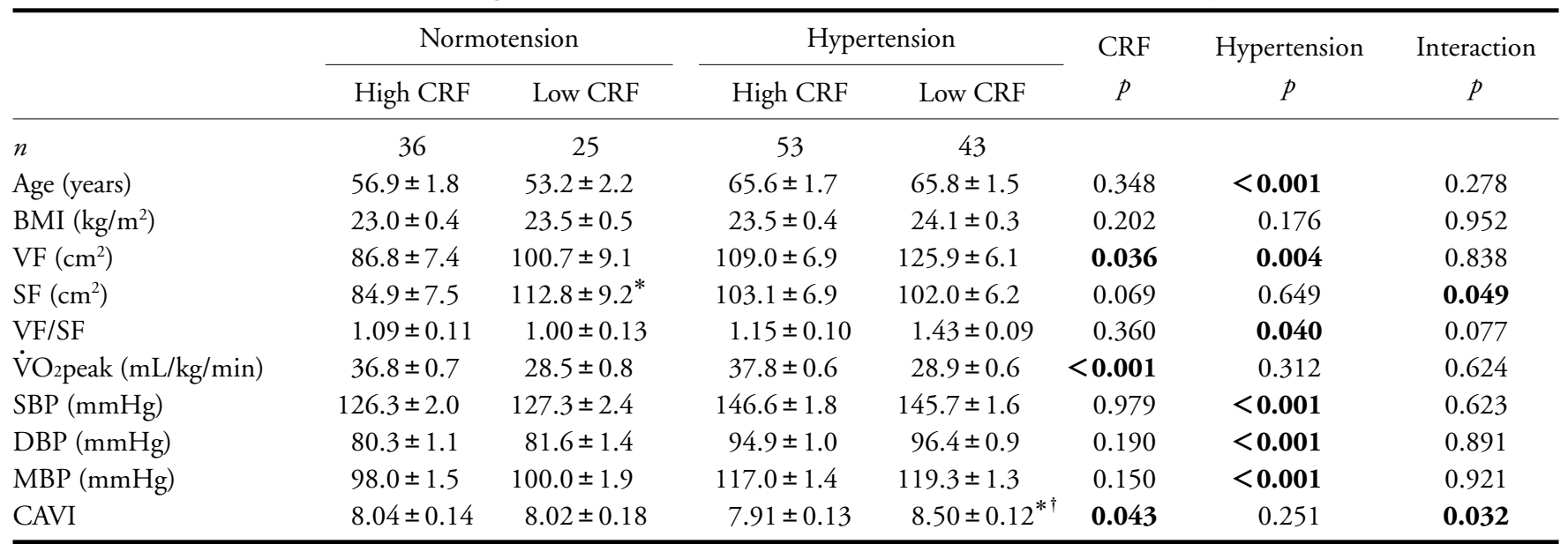

BMI: body mass index; VF: visceral fat; SF: subcutaneous fat; VF/SF: ratio of visceral fat to subcutaneous fat; VO$_{2}$ peak: peak oxygen uptake; SBP: systolic blood pressure; DBP: diastolic blood pressure; MBP: mean blood pressure; CAVI: cardio-ankle vascular index. The data are presented as the adjusted mean \pm SE and were analyzed using a two-way analysis of covariance adjusted for age. Boldface text indicates significance $(p<0.05) .{ }^{*} p<$ 0.05 vs. low CRF within the normotensive or hypertensive groups. $p<0.05$ vs. normotension within the same CRF group.

CRF groups of hypertensive subjects in their study $(n=61$, mean age: $57.9 \pm 14.3$ years). Because the blood pressure values did not differ according to the CRF levels in that study and the PWV is strongly influenced by blood pressure ${ }^{15,16)}$, the true relationship between CRF and arterial stiffness in hypertensive subjects may have been overlooked. In contrast, we assessed arterial stiffness by measuring the CAVI, which is theoretically independent of blood pressure ${ }^{18,19)}$. Notably, our data showed that, although the hypertensive individuals with a high CRF level had a low CAVI, the blood pressure values of these subjects did not differ from those of the hypertensive individuals with a low CRF (Table 4). This finding suggests that a low CAVI in hypertensive individuals with a high CRF is not accompanied by a decrease in blood pressure. Several epidemiological studies have demonstrated that hypertensive individuals with a high CRF exhibit a lower risk of CVD-related mortality, despite having comparable blood pressure values to those observed in low-CRF individuals ${ }^{27,28}$. Therefore, it is reasonable that a high CRF level is associated with reduced arterial stiffness independent of blood pressure. Hence, the methodological superiority of the CAVI may contribute to the identification of the true association between CRF and arterial stiffness, independent of blood pressure, in hypertensive individuals.

Although the mechanisms underlying our findings remain unknown, hypertension has been reported to induce arterial remodeling ${ }^{29)}$, which involves structural alternations in the arterial wall. In contrast, a high CRF has been shown to attenuate age-related arterial remodeling ${ }^{30)}$. Because age- and hypertensionrelated structural changes in the arterial wall are closely related to increased arterial stiffness ${ }^{31)}$, we speculate that a high CRF attenuates hypertension-related arterial stiffening in part by reducing arterial remodeling. Because the proportion of hypertensive subjects in the present study (mean age: $61.7 \pm 12.1$ years; hypertension: $61.1 \%$ ) is comparable to that of the age-matched general Japanese male population (60-69 years: $68.0 \%$; 70-79 years: $78.1 \%$; National Health and Nutrition Survey Japan, 2011, http://www.mhlw. go.jp/english/), the maintenance of a high CRF may attenuate arterial stiffening in most middle-aged and elderly Japanese men.

In contrast to that observed in the hypertensive individuals, the association between CRF and the CAVI was lost after adjusting for age in the normotensive individuals. A previous study failed to detect an independent association between CRF and arterial stiffening in healthy individuals, as noted in the present study ${ }^{10)}$. However, an age-independent significant association has also been reported ${ }^{6,11)}$. Fujie et al. ${ }^{32)}$ reported that the carotid $\beta$-stiffness values did not significantly differ according to the CRF levels in a healthy Japanese population $(n=837$, mean age: $44 \pm$ 1 years), whereas the $\beta$-stiffness values were elevated only in the low-CRF individuals with a specific genetic component. Therefore, it is possible that the normotensive subjects with a low CRF in the present study had genetic factors conferring resistance to arterial stiffness. Due to our limited sample size, further investigations using larger cohorts are required to clar- 
ify whether CRF is associated with the CAVI, even in normotensive individuals, regardless of their genetic predisposition.

Several studies have reported that, other than age, $\mathrm{VF}$ is the strongest predictor of the $\mathrm{CAVI}^{33,34)}$; however, the VF values were not associated with the CAVI independent of age and CRF in the present study (Table 3). Nagayama et al. ${ }^{33)}$ assessed the changes in the CAVI values in obese subjects who enrolled in a weight reduction program and reported that the improvement in the CAVI was mainly explained by a decrease in VF. Ohashi et al. ${ }^{34)}$ also demonstrated that the VF area is associated with the CAVI in middleaged healthy Japanese adults following adjustment for age. However, they did not evaluate the individual CRF levels, even though the CRF is inversely associated with the VF area and is an important determinant of arterial stiffening ${ }^{6,7)}$. Given the results of the current study, the observed association between the CAVI and VF in previous studies may have been mediated by CRF. Nevertheless, as many studies reported that visceral obesity is strongly associated with arterial stiffness and the incidence of $\mathrm{CVD}^{8,9,35)}$, further investigations with a large sample size are needed to draw conclusions on this issue.

We measured the blood lipid levels to determine whether the beneficial effect of a high CRF on the CAVI is mediated by an improvement in the blood lipid profile. In our study population, however, no blood lipid parameters were associated with the ageadjusted CAVI (Table 2). In concordance with these results, several studies have failed to detect a significant association between the CAVI and blood lipid levels ${ }^{36-38)}$. Higashiyama et al. ${ }^{36)}$ reported that the CAVI is more strongly correlated with the high-sensitivity C-reactive protein (hs-CRP) level than with the LDL cholesterol level. Furthermore, Masugata et al. ${ }^{37)}$ demonstrated that the CAVI is independently associated with the urinary 8-hydroxydeoxyguanosine (8-OHdG) level, but not any blood lipid parameters, in hypertensive patients. The hs-CRP and 8-OHdG levels are indicators of chronic inflammation and oxidative stress, respectively, and a high CRF is associated with low levels of these indicators ${ }^{39,40)}$. Therefore, the association between the CAVI and CRF is possibly mediated by a decrease in chronic inflammation and oxidative stress rather than improvements in blood lipid profiles.

The present study is associated with several limitations. First, our sample size was relatively small, which may have led to a type 2 error. Second, because this was a cross-sectional study, further prospective and interventional studies are needed to elucidate whether a high CRF significantly attenuates the agerelated increase in the CAVI. Third, our study population included only men. Women are well known to generally have less VF than men ${ }^{41)}$; therefore, the relationships between CRF, VF and the CAVI may differ according to sex. Further research is thus needed to confirm our findings in women.

\section{Conclusion}

In conclusion, the present study identified CRF to be a significant predictive factor of the CAVI, independent of age and VF, in hypertensive middle-aged and elderly Japanese men.

\section{Acknowledgments}

The authors would like to thank Hiroshi Kawano, Takafumi Ando and Taishi Susa for their valuable assistance.

\section{Notice of Grant Support}

This study was supported in part by a Grant-inAid for the Global COE (Sport Sciences for the Promotion of Active Life to Waseda University) from the Ministry of Education, Culture, Sports, Science and Technology (to M.H.) and a grant for strategic research initiatives (Paradigm shifts in a super-aged society) from Waseda University (to M.H.).

\section{Conflicts of Interest}

The authors do not have any conflicts of interest to disclose.

\section{References}

1) Avolio AP, Deng FQ, Li WQ, Luo YF, Huang ZD, Xing LF, Orourke MF: Effects of aging on arterial distensibility in populations with high and low prevalence of hypertension: comparison between urban and rural communities in China. Circulation, 1985; 71: 202-210

2) Tanaka H, Dinenno FA, Monahan KD, Clevenger CM, DeSouza CA, Seals DR: Aging, habitual exercise, and dynamic arterial compliance. Circulation, 2000; 102: $1270-1275$

3) Mattace-Raso FUS, van der Cammen TJM, Hofman A, van Popele NM, Bos ML, Schalekamp MADH, Asmar R, Reneman RS, Hoeks APG, Breteler MMB, Witteman JCM: Arterial stiffness and risk of coronary heart disease and stroke: the Rotterdam Study. Circulation, 2006; 113: 657-663

4) Blair SN, Kampert JB, Kohl HW, Barlow CE, Macera CA, Paffenbarger RS, Gibbons LW: Influences of cardio- 
respiratory fitness and other precursors on cardiovascular disease and all-cause mortality in men and women. JAMA, 1996; 276: 205-210

5) Wei M, Kampert JB, Barlow CE, Nichaman MZ, Gibbons LW, Paffenbarger RS Jr., Blair SN: Relationship between low cardiorespiratory fitness and mortality in normal-weight, overweight, and obese men. JAMA, 1999; 282: 1547-1553

6) Vaitkevicius PV, Fleg JL, Engel JH, Oconnor FC, Wright JG, Lakatta LE, Yin FCP, Lakatta EG: Effects of age and aerobic capacity on arterial stiffness in healthy-adults. Circulation, 1993; 88: 1456-1462

7) Wong SL, Katzmarzyk PT, Nichaman MZ, Church TS, Blair SN, Ross R: Cardiorespiratory fitness is associated with lower abdominal fat independent of body mass index. Med Sci Sport Exer, 2004; 36: 286-291

8) Orr JS, Gentile CL, Davy BM, Davy KP: Large artery stiffening with weight gain in humans: role of visceral fat accumulation. Hypertension, 2008; 51: 1519-1524

9) Sutton-Tyrrell K, Newman A, Simonsick EM, Havlik R, Pahor M, Lakatta E, Spurgeon H, Vaitkevicius P, Investigators HA: Aortic stiffness is associated with visceral adiposity in older adults enrolled in the study of health, aging, and body composition. Hypertension, 2001; 38: 429-433

10) Christou DD, Gentile CL, DeSouza CA, Seals DR, Gates PE: Fatness is a better predictor of cardiovascular disease risk factor profile than aerobic fitness in healthy men. Circulation, 2005; 111: 1904-1914

11) Boreham CA, Ferreira I, Twisk JW, Gallagher AM, Savage MJ, Murray LJ: Cardiorespiratory fitness, physical activity, and arterial stiffness: the Northern Ireland Young Hearts Project. Hypertension, 2004; 44: 721-726

12) AlGhatrif M, Strait JB, Morrell CH, Canepa M, Wright J, Elango P, Scuteri A, Najjar SS, Ferrucci L, Lakatta EG: Longitudinal Trajectories of arterial stiffness and the role of blood pressure: the Baltimore Longitudinal Study of Aging. Hypertension, 2013; 62: 934-941

13) Benetos A, Adamopoulos C, Bureau JM, Temmar M, Labat C, Bean K, Thomas F, Pannier B, Asmar R, Zureik M, Safar M, Guize L: Determinants of accelerated progression of arterial stiffness in normotensive subjects and in treated hypertensive subjects over a 6-year period. Circulation, 2002; 105: 1202-1207

14) Yamashina $A$, Tomiyama $H$, Takeda $K$, Tsuda $H$, Arai $T$, Hirose K, Koji Y, Hori S, Yamamoto Y: Validity, reproducibility, and clinical significance of noninvasive brachial-ankle pulse wave velocity measurement. Hypertens Res, 2002; 25: 359-364

15) Ito N, Ohishi M, Takagi T, Terai M, Shiota A, Hayashi N, Rakugi H, Ogihara T: Clinical usefulness and limitations of brachial-ankle pulse wave velocity in the evaluation of cardiovascular complications in hypertensive patients. Hypertens Res, 2006; 29: 989-995

16) Matsui Y, Kario K, Ishikawa J, Eguchi K, Hoshide S, Shimada K: Reproducibility of arterial stiffness indices (pulse wave velocity and augmentation index) simultaneously assessed by automated pulse wave analysis and their associated risk factors in essential hypertensive patients. Hypertens Res, 2004; 27: 851-857

17) Yamashina A, Tomiyama $H$, Arai T, Koji Y, Yambe M,
Motobe H, Glunizia Z, Yamamoto Y, Hori S: Nomogram of the relation of brachial-ankle pulse wave velocity with blood pressure. Hypertens Res, 2003; 26: 801-806

18) Kubozono T, Miyata M, Ueyama K, Nagaki A, Otsuji Y, Kusano K, Kubozono O, Tei C: Clinical significance and reproducibility of new arterial distensibility index. Circ J, 2007; 71: 89-94

19) Shirai K, Utino J, Otsuka K, Takata M: A novel blood pressure-independent arterial wall stiffness parameter; cardio-ankle vascular index (CAVI). J Atheroscler Thromb, 2006; 13: 101-107

20) Kobayashi S, Honda S, Murakami K, Sasaki S, Okubo H, Hirota N, Notsu A, Fukui M, Date C: Both comprehensive and brief self-administered diet history questionnaires satisfactorily rank nutrient intakes in Japanese adults. J Epidemiol, 2012; 22: 151-159

21) Aoyama T, Asaka M, Ishijima T, Kawano H, Cao ZB, Sakamoto S, Tabata I, Higuchi M: Association between muscular strength and metabolic risk in Japanese women, but not in men. J Physiol Anthropol, 2011; 30: 133-139

22) Kawano $H$, Iemitsu M, Gando $Y$, Ishijima $T$, Asaka $M$, Aoyama T, Ando T, Tokizawa K, Miyachi M, Sakamoto S, Higuchi M: Habitual rowing exercise is associated with high physical fitness without affecting arterial stiffness in older men. J Sport Sci, 2012; 30: 241-246

23) Lindholm H, Punakallio A, Lusa S, Sainio M, Ponocny E, Winker R: Association of cardio-ankle vascular index with physical fitness and cognitive symptoms in aging Finnish firefighters. Int Arch Occ Env Hea, 2012; 85: 397-403

24) Fitzgerald MD, Tanaka H, Tran ZV, Seals DR: Agerelated declines in maximal aerobic capacity in regularly exercising vs. sedentary women: A meta-analysis. J Appl Physiol, 1997; 83: 160-165

25) Fleg JL, Morrell CH, Bos AG, Brant LJ, Talbot LA, Wright JG, Lakatta EG: Accelerated longitudinal decline of aerobic capacity in healthy older adults. Circulation, 2005; 112: 674-682

26) Kraft KA, Arena R, Arrowood JA, Fei DY: High aerobic capacity does not attenuate aortic stiffness in hypertensive subjects. Am Heart J, 2007; 154: 976-982

27) Church TS, Kampert JB, Gibbons LW, Barlow CE, Blair $\mathrm{SN}$ : Usefulness of cardiorespiratory fitness as a predictor of all-cause and cardiovascular disease mortality in men with systemic hypertension. Am J Cardiol, 2001; 88: 651656

28) Sui XM, LaMonte MJ, Blair SN: Cardiorespiratory fitness and risk of nonfatal cardiovascular disease in women and men with hypertension. Am J Hypertens, 2007; 20: 608615

29) Intengan HD, Schiffrin EL: Vascular remodeling in hypertension: roles of apoptosis, inflammation, and fibrosis. Hypertension, 2001; 38: 581-587

30) Gando Y, Yamamoto K, Kawano H, Murakami H, Ohmori Y, Kawakami R, Sanada K, Higuchi M, Tabata I, Miyachi M: Attenuated age-related carotid arterial remodeling in adults with a high level of cardiorespiratory fitness. J Atheroscler Thromb, 2011; 18: 248-254

31) Dao HH, Essalihi R, Bouvet C, Moreau P: Evolution and modulation of age-related medial elastocalcinosis: impact on large artery stiffness and isolated systolic hypertension. 
Cardiovasc Res, 2005; 66: 307-317

32) Fujie $S$, Iemitsu M, Murakami H, Sanada K, Kawano H, Gando Y, Kawakami R, Miyachi M: Higher cardiorespiratory fitness attenuates arterial stiffening associated with the Ala54Thr polymorphism in FABP2. Physiol Genomics, 2013; 45: 237-242

33) Nagayama D, Endo K, Ohira M, Yamaguchi T, Ban N, Kawana H, Nagumo A, Saiki A, Oyama T, Miyashita Y, Shirai K: Effects of body weight reduction on cardioankle vascular index (CAVI). Obes Res Clin Pract, 2013; 7: E139-E145

34) Ohashi N, Ito C, Fujikawa R, Yamamoto H, Kihara $Y$, Kohno N: The impact of visceral adipose tissue and highmolecular weight adiponectin on cardio-ankle vascular index in asymptomatic Japanese subjects. Metabolism, 2009; 58: 1023-1029

35) Van Gaal LF, Mertens IL, De Block CE: Mechanisms linking obesity with cardiovascular disease. Nature, 2006; 444: $875-880$

36) Higashiyama A, Wakabayashi I, Kubota Y, Adachi Y, Hayashibe A, Nishimura K, Sugiyama D, Kadota A, Imano $\mathrm{H}$, Miyamatsu N, Miyamoto Y, Okamura T: Does high-sensitivity C-reactive protein or low-density lipoprotein cholesterol show a stronger relationship with the car- dio-ankle vascular index in healthy community dwellers?: the KOBE Study. J Atheroscler Thromb, 2012; 19: 10271034

37) Masugata $H$, Senda $S$, Murao K, Inukai $M$, Himoto $T$, Hosomi N, Okada H, Goda F: Association between urinary 8-hydroxydeoxyguanosine, an indicator of oxidative stress, and the cardio-ankle vascular index in hypertensive patients. J Atheroscler Thromb, 2012; 19: 747-755

38) Soska V, Frantisova M, Dobsak P, Dusek L, Jarkovsky J, Novakova M, Shirai K, Fajkusova L, Freiberger T: Cardioankle vascular index in subjects with dyslipidaemia and other cardiovascular risk factors. J Atheroscler Thromb, 2013; 20: 443-451

39) Church TS, Barlow CE, Earnest CP, Kampert JB, Priest EL, Blair SN: Associations between cardiorespiratory fitness and C-reactive protein in men. Arterioscl Throm Vas, 2002; 22: 1869-1876

40) Traustadottir T, Davies SS, Su YL, Choi L, Brown-Borg HM, Roberts LJ, Harman SM: Oxidative stress in older adults: effects of physical fitness. Age, 2012; 34: 969-982

41) Lemieux S, Prudhomme D, Bouchard C, Tremblay A, Despres JP: Sex-differences in the relation of visceral adipose-tissue accumulation to total-body fatness. Am J Clin Nutr, 1993; 58: 463-467 